\title{
Radiation safety summit - method to estimate radiation dose to pediatric patients from CT scans
}

\author{
Keith J. Strauss
}

(C) Springer-Verlag 2011

State-of-the-art CT scanners typically display two dose indices, $\mathrm{CTDI}_{\mathrm{vol}}(\mathrm{mGy})$ and DLP (mGy-cm), along with which two standard CTDI phantoms, 16- or 32-cm diameter, are used to estimate the two indices. $\mathrm{CTDI}_{\mathrm{vol}}$ is based on radiation dose measurements on an individual scanner completed by a medical physicist with a pencil ionization chamber and either a $16-$ or $32-\mathrm{cm}$ diameter CTDI cylindrical plastic phantom.

$\mathrm{CTDI}_{\mathrm{vol}}$ represents the radiation dose delivered to a standard plastic phantom from a specific model CT scanner using specified scan parameters: tube voltage, tube current, rotation time, pitch and selected bowtie filter. $\mathrm{CTDI}_{\mathrm{vol}}$ provides a standardized method to estimate and compare the radiation output of different $\mathrm{CT}$ scanners. $\mathrm{CTDI}_{\mathrm{vol}}$ is defined to be a dose index of CT scanners, not an indication of patient dose.

The inability of $\mathrm{CTDI}_{\mathrm{vol}}$ to accurately reflect pediatric patient dose is illustrated in Fig. 1. Three cylindrical phantoms with diameters of $10 \mathrm{~cm}, 16 \mathrm{~cm}$, and $32 \mathrm{~cm}$ (representing the smallest pediatric patient to an adult) were scanned with the same scanner using the same scan parameters. While this delivered the same radiation energy to each phantom, the measured $\mathrm{CTDI}_{\mathrm{vol}}$ in the phantoms (top row of data in figure) ranged from $47 \mathrm{mGy}$ to $18 \mathrm{mGy}$. This clearly indicates that the dose to various-size patients (represented by the three phantoms) is a function of the energy produced by the scanner (determined by scan techniques) and the size of the patient. The superficial tissues of the large patient attenuate the radiation beam,

Disclaimer Dr. Strauss has no financial interests, investigational or off-label uses to disclose.

K. J. Strauss $(\bowtie)$

Children's Hospital Boston, Harvard Medical School,

Boston, MA 02115, USA

e-mail: Keith.Strauss@TCH.harvard.edu reducing the dose delivered to the core of the large patient. In the figure, the center of the $10-\mathrm{cm}$ phantom received 47 mGy of dose while the center of the $32-\mathrm{cm}$ phantom received a dose of only $10.8 \mathrm{mGy}$ for the same scan techniques.

While the measured dose index in the previous example with an ionization chamber, $\mathrm{CTDI}_{\mathrm{vol}}$, is very dependent on patient size when scan parameters for all three phantoms are identical, the displayed $\mathrm{CTDI}_{\mathrm{vol}}$ on a CT scanner is independent of changes in patient size by definition. The displayed scan index assumes the actual scan parameters but by definition assumes the patient is either a $16-\mathrm{cm}$ or $32-\mathrm{cm}$ diameter cylindrical plastic phantom. If the $16-\mathrm{cm}$ phantom is assumed, the scanner will display $37 \mathrm{mGy}$ for $\mathrm{CTDI}_{\mathrm{vol}}$ for each of the three represented patient sizes (second row of data in Fig. 1); this overestimates the dose to adult patients and underestimates dose to infants. If the $32-\mathrm{cm}$ phantom is assumed, the scanner displays $18 \mathrm{mGy}$ for all patients (third row of data in figure); this underestimates the dose to all patients smaller than adults. In either case, the displayed $C T D I_{v o l}$ does not represent the pediatric patient dose and can mislead the technologist or radiologist.

A task group of the American Association of Physicists in Medicine (AAPM), co-chaired by John Boone and Keith Strauss, is currently preparing a report that will provide scaling factors as a function of patient size that can be used by the technologist or radiologist to more accurately estimate the radiation dose to the tissues of the pediatric patient that are directly irradiated by the primary X-ray beam of the CT scanner. These scaling factors will be based on attenuation data collected from numerous CT scanners by multiple investigators. Hopefully, these scaling factors will be available by the time of this presentation.

These scaling factors should allow the operator of the CT scanner to estimate the radiation dose to the 
Fig. 1 Measured versus displayed $\mathrm{CTDI}_{\mathrm{vol}}$ values

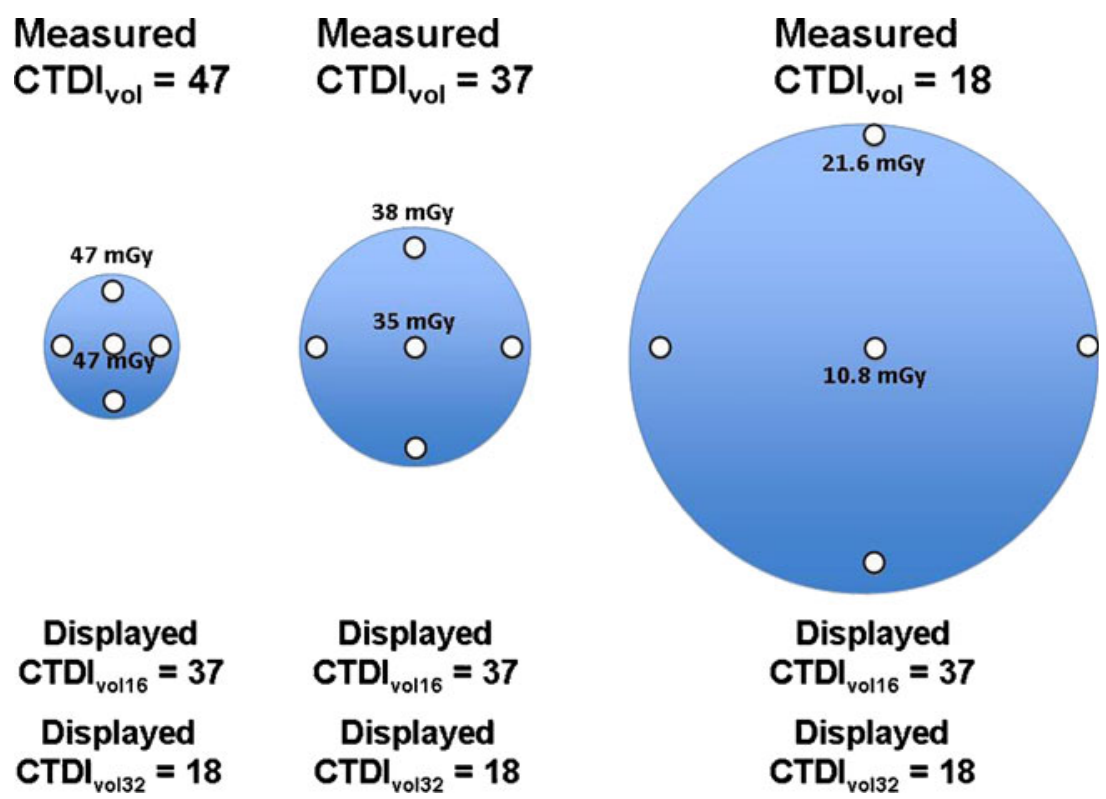

pediatric patient after the projection scan is complete, but prior to acquiring the scan data. If the estimated patient dose is outside an expected range, the operator has the opportunity to appropriately adjust scan parameters prior to delivering the vast majority of the radiation dose of the CT examination. To estimate the pediatric patient dose, the operator must measure the scan width of the patient either with mechanical calipers when positioning the patient or graphically on the image of the projection scan. In addition, the displayed CTDI $_{\mathrm{vol}}$ (after scan projection) and phantom size it is based upon must be noted by the operator. Knowing patient size, $\mathrm{CTDI}_{\mathrm{vol}}$ and phantom size allows the operator to select the correct scaling factor and multiply it by the $\mathrm{CTDI}_{\mathrm{vol}}$ with a simple handheld calculator to estimate the pediatric patient dose. 